\title{
Central-Variant Posterior Reversible Encephalopathy Syndrome with Albuminocytologic Dissociation
}

\author{
Sang-Woo Lee Seung-Jae Lee \\ Department of Neurology, Soonchunhyang University Bucheon Hospital, \\ Bucheon, South Korea
}

\section{Keywords}

Encephalopathy · Brain stem · Central zone · Albuminocytologic dissociation · Cerebrospinal fluid

\begin{abstract}
Posterior reversible encephalopathy syndrome (PRES) is a disorder of reversible vasogenic brain edema which mainly involves the parieto-occipital lobes in various clinical settings. The main mechanism is known to be cerebral autoregulation failure and endothelial dysfunction leading to the disruption of the blood-brain barrier. We report the case of a 47-year-old woman with PRES which involved the brain stem and thalami, sparing the cerebral hemispheres. She was admitted to the emergency room because of acute-onset confusion. Her initial blood pressure was $270 / 220 \mathrm{~mm} \mathrm{Hg}$. Routine blood lab tests showed pleocytosis, hyperglycemia, and azotemia. Brain magnetic resonance imaging (MRI) showed a lesion of vasogenic edema involving nearly the whole area of pons, the left side of the midbrain, and the bilateral medial thalami. Cerebrospinal fluid (CSF) examination revealed an increased level of protein with normal white blood cell count. With conservative care, the patient markedly recovered 3 days after symptom onset, and a follow-up MRI confirmed complete resolution of the vasogenic edema. This case suggests that PRES can rarely involve the "central zone" only, sparing the cerebral hemispheres, which may be confused with other neurological diseases. Besides, the CSF albuminocytologic dissociation may suggest the disruption of the blood-brain barrier in patients with PRES.

(C) 2018 The Author(s)

Published by S. Karger AG, Basel
\end{abstract}

KARGER

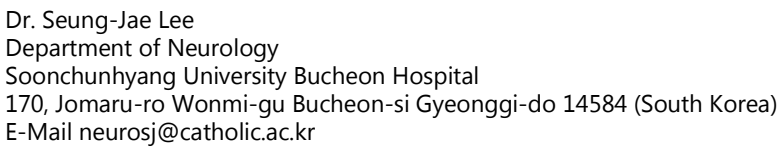




\section{Case Reports in Neurology}

Lee and Lee: Central-Variant Posterior Reversible Encephalopathy Syndrome with Albuminocytologic Dissociation

\section{Introduction}

Posterior reversible encephalopathy syndrome (PRES) is a disorder of vasogenic brain edema in the setting of renal failure, uncontrolled hypertension, cytotoxic agents, autoimmune disorders, and pre-eclampsia or eclampsia [1]. It usually involves the bilateral cerebral hemispheres, especially the parieto-occipital lobes as a typical lesion location with or without involvement of the central zone (basal ganglia, brain stem, and cerebellum) [2]. Isolated involvement of the central zone is rare, and thus prompts the need for further differential diagnosis [1-3].

Data on findings of cerebrospinal fluid (CSF) in patients with PRES are few in the literature to date, though 2 recent studies reported albuminocytologic dissociation as the most common CSF profile in PRES [4, 5]. We herein report a case of PRES which involved the brain stem and thalami, sparing the cerebral hemispheres, and showed albuminocytologic dissociation.

\section{Case Presentation}

A 47-year-old, previously healthy woman was admitted to the emergency room because of acute-onset confusion occurring in the morning of the admission day. Her blood pressure at presentation was $270 / 220 \mathrm{~mm} \mathrm{Hg}$, her heart rate was 112 beats per minute, and her body temperature was $38.1^{\circ} \mathrm{C}$. Neurological examination revealed disorientation to time, place, and person, and bilateral positive Babinski responses, but there was no other focal sign. Routine laboratory tests showed a peripheral blood pleocytosis (leukocytes $14,010 / \mathrm{uL}$ ), azotemia (blood urea nitrogen $48.7 \mathrm{mg} / \mathrm{dL}$, creatinine $3.0 \mathrm{mg} / \mathrm{dL}$ ), and hyperglycemia (fasting blood sugar $282 \mathrm{mg} / \mathrm{dL} ; \mathrm{HbA}_{1 \mathrm{c}}$ 7.3\%). Serum sodium was $134 \mathrm{mmol} / \mathrm{L}$. The high-sensitivity C-reactive protein level was slightly increased $(0.83 \mathrm{mg} / \mathrm{dL}$ [range $0-0.5 \mathrm{mg} / \mathrm{dL}])$. Other laboratory findings including immunologic tests were normal: ammonia $36 \mathrm{ug} / \mathrm{dL}$ (range 15-45 ug/dL), anti-nuclear antibody (-), anti-phospholipid antibody immunoglobulin G/ immunoglobulin $\mathrm{M}(-/-)$, rheumatoid factor $5 \mathrm{IU} / \mathrm{mL}$ (range, $0-18 \mathrm{IU} / \mathrm{mL}$ ), and lupus anticoagulant (-).

Brain magnetic resonance imaging (MRI) revealed a lesion of vasogenic edema without enhancement involving nearly the whole area of pons, the left side of the midbrain, and the bilateral medial thalami (Fig. 1a-c). Diffusion-weighted and gradient-echo MRI were unremarkable. Electroencephalogram showed continuous attenuation of background activity with loss of posterior dominant rhythm. Spinal tap was conducted. Opening pressure was slightly increased $\left(21 \mathrm{~cm} \mathrm{H}_{2} \mathrm{O}\right)$. The levels of CSF protein $(102.6 \mathrm{mg} / \mathrm{dL})$ and albumin $(64.8$ $\mathrm{mg} / \mathrm{dL}$ ) were high (range 15-45 mg/dL). However, other CSF profiles were normal: red blood cells were absent, leukocyte count $4 / \mathrm{mm}^{3}$, glucose $159.3 \mathrm{mg} / \mathrm{dL}$ (serum glucose 267 $\mathrm{mg} / \mathrm{dL}$ ), and oligoclonal band negative.

Fever subsided spontaneously without a specific treatment within 1 day. No bacterial species were recovered from cultures of 3 blood samples. Urinalysis did not show bacteriuria or pyuria, and urine culture was negative for bacteria. CSF polymerase chain reaction tests for herpes simplex virus type I and II, varicella zoster virus, cytomegalovirus, EpsteinBarr virus, and Japanese encephalitis virus were all negative. In addition, sputum gram stain, bacterial culture, and smear for acid-fast bacilli were negative. Therefore, the patient was treated only conservatively for hypertension, azotemia, and hyperglycemia. The patient's symptoms completely resolved 3 days after symptom onset, and the edema was 


\section{Case Reports in Neurology}

Lee and Lee: Central-Variant Posterior Reversible Encephalopathy Syndrome with Albuminocytologic Dissociation

confirmed to have disappeared almost completely on the MRI conducted on the ninth hospital day (Fig. 1d-f).

\section{Discussion}

The case, typically as in other cases, presented acute-onset encephalopathy with cerebral vasogenic edema in high-risk conditions for PRES (severe hypertension and renal failure). However, it is unusual in terms of the isolated involvement of the central zone (brain stem and thalami), sparing the cerebral hemispheres mostly involved by PRES. In particular, the parieto-occipital lobes are known to be the most prevalent regions of PRES [1, 2]. It may be attributable to a relative lack of sympathetic innervation in the regions, and thus uncontrolled increase of blood perfusion leading to cerebral edema [6].

Like our case, atypical lesion locations have rarely been reported in patients with PRES [3]. Especially, a lesion affecting solely the "central zone" necessitates clinicians to differentially diagnose it from other potential neurological disorders [1]. These include osmotic demyelination syndrome, which usually occurs in the setting of rapid correction of hyponatremia, alcohol poisoning, and organ transplantation. The lesion mainly occurs in pons, where it is often seen as a bat-wing appearance on MRI because of its preferential involvement of the central part of pons with preservation of the outer part [1]. Besides, central nervous system (CNS) infection should be excluded if fever is present like in our case. Thus, spinal tapping was performed in our case to exclude CNS infection. CNS vasculitis was also excluded from diagnosis through laboratory studies.

Another peculiar finding in this case was albuminocytologic dissociation in CSF. Even so, most of the prior case studies did not mention CSF findings in patients with central-variant PRES $[3,7]$, whereas only a few reports described normal CSF profiles [8, 9]. This is probably because a CSF finding may not be mandatory for its diagnosis. Meanwhile, 2 small-size studies have recently suggested that albuminocytologic dissociation may be the most common CSF profile in PRES $[4,5]$. The finding may reflect the underlying pathogenic mechanism of PRES with blood-brain barrier disruption due to dysfunction of cerebral autoregulation and small vessel endothelium. Notably, a study showed that the brain central zone may be associated with higher elevation of CSF protein in line with the finding in our case [4]. This could be explained by the regional vulnerability for increase in small vessel permeability, causing increased leakage of protein into the extravascular space under hypertensive conditions. The assumption can be supported by the fact that the central zone is a frequent region for hypertensive intracerebral hemorrhage.

\section{Conclusion}

Our case was peculiar because of the atypical, central-zone location of the lesion and CSF albuminocytologic dissociation in PRES. Atypical lesion location can be challenging in the diagnosis of PRES. Accordingly, clinicians should consider the clinical context in patients with suspicion of PRES, in addition to radiologic findings. Albuminocytologic dissociation in CSF may be found in PRES, but its diagnostic value for PRES should be clarified in a largescale future study. 
Lee and Lee: Central-Variant Posterior Reversible Encephalopathy Syndrome with Albuminocytologic Dissociation

\section{Statement of Ethics}

No ethics approval was needed for this case report. The patient gave informed consent for publication of his clinical details.

\section{Disclosure Statement}

The present authors declare that they have no competing interests.

\section{Funding Sources}

This work was supported by the Soonchunhyang University Research Fund.

\section{Author Contributions}

S.-J.L. conceived and designed the study and revised the manuscript. He also created the figure. S.-W.L. participated in the design of the study and wrote the manuscript. All authors contributed to data acquisition and interpretation as well as the final drafts of the manuscript. All authors read and approved the final manuscript.

\section{References}

1 Fugate JE, Rabinstein AA: Posterior reversible encephalopathy syndrome: clinical and radiological manifestations, pathophysiology, and outstanding questions. Lancet Neurol 2015;14:914-925.

-2 McKinney AM, Short J, Truwit CL, et al: Posterior reversible encephalopathy syndrome: incidence of atypical regions of involvement and imaging findings. Am J Roentgenol 2007;189:904-912.

-3 McKinney AM, Jagadeesan BD, Truwit CL: Central-variant posterior reversible encephalopathy syndrome: brainstem or basal ganglia involvement lacking cortical or subcortical cerebral edema. Am J Roentgenol 2013;201:631-638.

4 Datar S, Singh TD, Fugate JE, et al: Albuminocytologic dissociation in posterior reversible encephalopathy syndrome. Mayo Clin Proc 2015;90:1366-1371.

-5 Neeb L, Hoekstra J, Endres M, et al: Spectrum of cerebral spinal fluid findings in patients with posterior reversible encephalopathy syndrome. J Neurol 2016;263:30-34.

-6 Sheth RD, Riggs JE, Bodenstenier JB, et al: Parietal occipital edema in hypertensive encephalopathy: a pathogenic mechanism. Eur Neurol 1996;36:25-28.

-7 Srinivasan KG, Balasubramanian P, Mayilvaganan KR, et al: Central variant of posterior reversible encephalopathy syndrome - a rare case report. J Clin Diagn Res 2017;11:TD01-TD02.

-8 Ocek L, Sener U, Demirtas BS, et al: Central-variant posterior reversible encephalopathy due to sulfasalazine: a case report. Med Princ Pract 2015;24:578-580.

\$9 Gao B, Liang H, Liu FL, et al: Isolated pons involvement in posterior reversible encephalopathy syndrome in a patient with chronic renal insufficiency: case report and literature review. Clin Neuroradiol 2012;22:341-344. 


\section{Case Reports in Neurology}

\begin{tabular}{l|l}
\hline Case Rep Neurol 2018;10:29-33 \\
\hline DOI: 10.1159/000486444 & $\begin{array}{l}\text { C 2018 The Author(s). Published by S. Karger AG, Basel } \\
\text { www.karger.com/crn }\end{array}$ \\
\hline
\end{tabular}

Lee and Lee: Central-Variant Posterior Reversible Encephalopathy Syndrome with Albuminocytologic Dissociation
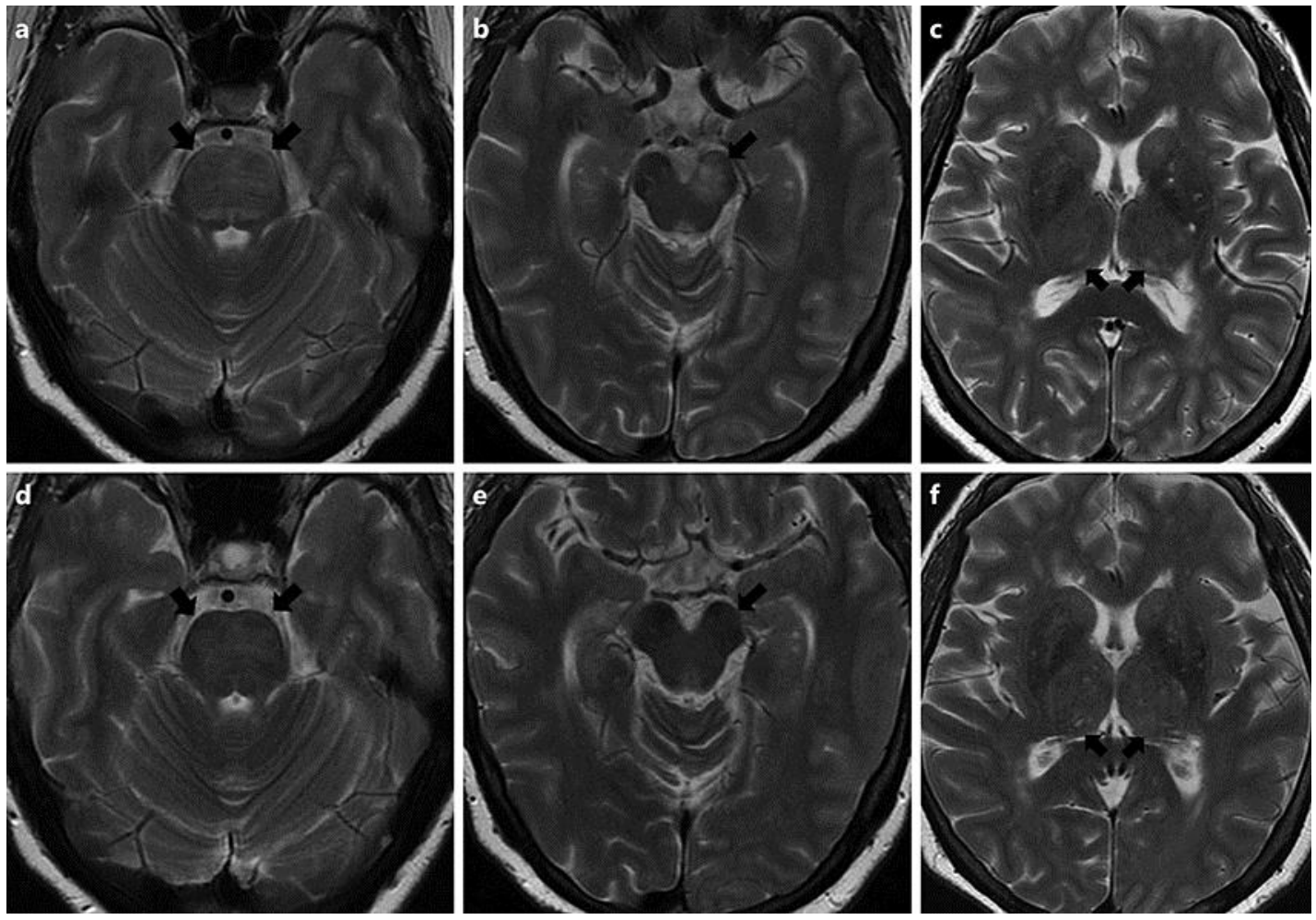

Fig. 1. T2-weighted brain magnetic resonance image (MRI) conducted at presentation revealed high-signal lesions (black arrows) in nearly the whole area of pons (a), the left side of the midbrain (b), and the bilateral medial thalami (c). These lesions disappeared almost completely on MRI conducted on the ninth hospital day $(\mathbf{d}-\mathbf{f})$. 